\title{
A Group Decision Matrix Construction Method Based On OWA Operator
}

\author{
Yanling Li ${ }^{1, a}$, Qifei Yan ${ }^{2, b}$, Xinli Yin ${ }^{1, a}$ \\ ${ }^{1}$ Xi'an Hong Qing High Tech Research Institute, Xi'an, 710025, China \\ ${ }^{2}$ Xi'an Communication Institute, Xi'an, 710106, China \\ aemail:Iyling998@163.com, bemail:732613497@qq.com
}

Keywords: Group Decision; OWA Operator; Experts Weight; Group Decision Matrix;

\begin{abstract}
A method of constructing a group decision matrix based on OWA operator is proposed for the construction method of the group decision matrix research. In this method, the OWA operator is used to aggregate the expert's decision matrix into the initial group decision matrix, then the degree of deviation from the expert's decision matrix and the group decision matrix is obtained base on distance measure so as to determine the weights of experts, finally, the group decision matrix is obtained by the weighted arithmetic average operator. Numerical results show that the quality of group decision matrix constructed by method of this paper is relatively high, compared to the decision matrix constructed by usual method of arithmetic mean, geometric mean and OWA operator.
\end{abstract}

\section{Introduction}

Multi attribute decision making is widely used in the fields of economy, management, military and engineering technology. With the development of information technology and the increasing complexity of decision-making problems, more and more problems are solved in the form of the group decision-making.

With respect to the general multi-attribute decision-making problems, the key problem of group decision making is to integrate the individual evaluation opinion of the expert to a group opinion that all of them relatively satisfy or vote for. However, because of the differences in cognitive level, evaluation experience and personal preference of the experts, their evaluation opinions are not entirely consistent. On this problem the solution proposed by relevant literatures is to assign different weights between experts, so as to restrain the influence of the individual expert opinion which is so much different from group opinion. Thus, there are two main concerns in the research on the aggregation of individual expert opinion: one is the method for expert opinion aggregation [1][2][3]; the other is the method to determine the experts' weights [4][5].But there is no method which is recognized as the best method. Therefore, it is necessary to study the method of expert opinion aggregation based on the actual decision needs and the specific form of decision information.

Decision matrix is one of the important decision-making information form in multiple attribute decision making, and its quality is one of the basic conditions for ensuring the objective and reasonable results of multiple attribute decision making. In view of the construction of group decision matrix, a group decision-making matrix construction method based on OWA operator(Ordered Weighted Averaging Operator) has been proposed in this paper, The main idea is: firstly, aggregate the expert decision matrix into the initial group decision matrix by using the OWA operator, and then analyze the deviation between the individual decision matrix and the group decision matrix by a certain method, decide experts' weight based on according to the degree of deviation, the expert with large degree of deviation will be given less weight, and then a group decision matrix with higher degree of consistency will be obtained by using weighted arithmetic mean method. 


\section{OWA Operator}

OWA operator is proposed by Professor Yager, because it has some good characteristics, and is easy to understand and calculate. So,it has many successful applications in group decision analysis, mathematical programming and so on. It is widely considered as an effective method for information integration.

Definition: Let $f: R^{n} \rightarrow R, f_{B}\left(x_{1}, x_{2}, \cdots, x_{n}\right)=\sum_{i=1}^{n} \alpha_{i} x_{i}^{\prime}$ as a function with $n$ elements, $x_{i}^{\prime}$ is the $i$-th largest element in $\left(x_{1}, x_{2}, \cdots, x_{n}\right), \alpha=\left(\alpha_{1}, \alpha_{2}, \cdots, \alpha_{n}\right)^{T}$ function is a weighted vector associated with $f$,and $\alpha_{i} \in[0,1], \sum_{i=1}^{n} \alpha_{i}=1$, then $f$ is called $n$-dimensional ordered weighted averaging operator(OWA).

The generating method of weight vector $\alpha=\left(\alpha_{1}, \alpha_{2}, \cdots, \alpha_{n}\right)^{T}$ of OWA operator has many kinds of methods, such as the fitting method, planning method, substitution method, function method, and so on [2]. In which fuzzy function method is widely used because of it's better description for words such as "at least half", "most", "as much as possible " and so on. Commonly used fuzzy quantization operator, is shown as follows:

$$
Q(x)= \begin{cases}0 & , x \leq a \\ \frac{x-a}{b-a} & , a<x<b, a 、 b \in[0,1] \\ 1 & , x \leq b\end{cases}
$$

In the type: the parameters $(a, b)$ of fuzzy Quantifying operator $Q(x)$ are $(0,0.5),(0.3,0.8),(0.5,1)$ under the principle of "at least half", "most" and "as much as possible". Then the operator weight vector of OWA operator is calculated as follows:

$\alpha_{i}=Q\left(\frac{i}{n}\right)-Q\left(\frac{i-1}{n}\right)$

For example, When $X=\left(x_{1}, x_{2}, \cdots, x_{5}\right)$, then the $\alpha=\left(\alpha_{1}, \alpha_{2}, \cdots, \alpha_{5}\right)^{T}$ is shown as Fig.1,it is clearly shows the selecting tendency under different principle.

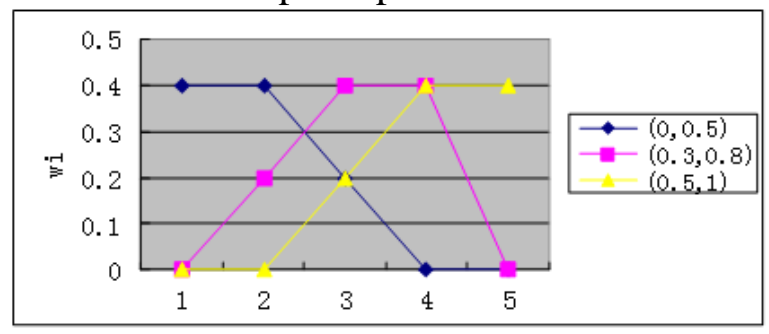

Fig.1. Weight vector of OWA operator

\section{Group Decision Matrix Construction Method Based on OWA Operator}

Let $A=\left\{A_{1}, A_{2}, \cdots, A_{m}\right\}$ as a decision scheme set for decision problem, $C=\left\{C_{1}, C_{2}, \cdots, C_{m}\right\}$ as a set consisted of $n$ evaluation index (or attribute), the decision making group $E=\left\{e_{1}, e_{2}, \cdots, e_{l}\right\}$ is consisted of $l$ decision maker, the individual expert decision matrix $V^{k}=\left[v_{i j}^{k}\right]_{m \times n}$ will be got, is shown as follows:

$$
V^{k}=\left[\begin{array}{cccc}
v_{11}^{k} & v_{12}^{k} & \cdots & v_{1 n}^{k} \\
v_{21}^{k} & v_{22}^{k} & \cdots & v_{2 n}^{k} \\
\cdots & \cdots & \cdots & \cdots \\
v_{m 1}^{k} & v_{m 2}^{k} & \cdots & v_{m n}^{k}
\end{array}\right] \quad(k=1,2, \cdots, l)
$$

Weight of each expert is determined according to the deviation between individual evaluation 
opinion he gives and group opinion at this evaluation problem, expressed as $B=\left\{\beta_{1}, \beta_{2}, \cdots \beta_{l}\right\}, \sum_{k=1}^{l} \beta_{k}=1$. Finally, the individual decision matrix $V^{k}=\left[v_{i j}^{k}\right]_{m \times n}$ is weighted by expert weight, and then it is integrated to group decision matrix $V^{q}=\left[v_{i j}^{q}\right]_{m \times n}$.

When building group decision matrix, because we do not know the extent of deviation between the individual decision matrix and the group decision matrix, so if group decision matrix is directly calculated through arithmetic average, the index value of group decision matrix is possible influenced by its maximum or minimum value, therefore the group opinion tend to opinion of experts that gives the index very high evaluation or very low evaluation. So this paper integrate individual decision matrix with OWA operator to get a initial group decision matrix that represent most of the expert.

For $v_{i j}^{k}$ of individual decision matrix $V^{k}=\left[v_{i j}^{k}\right]_{m \times n}$ from experts, construct The initial group decision matrix is recorded as $V^{c}=\left[V_{i j}^{c}\right]_{m \times n}$, the element $v_{i j}^{c}$ is as follows: $v_{i j}^{c}=O W A\left(v_{i j}^{1}, v_{i j}^{2}, \cdots, v_{i j}^{l}\right)=\sum_{k=1}^{l} \alpha_{k} x_{i j}^{k}$

In the type, $x_{i j}^{k}$ is the is the element ranked $k$ in $\left(v_{i j}^{1}, v_{i j}^{2}, \cdots, v_{i j}^{l}\right)$, the weight vector $\alpha=\left(\alpha_{1}, \alpha_{2}, \cdots, \alpha_{n}\right)^{T}$ use fuzzy quantization operator formula, namely calculated according to Eq.2and Eq.3. Because we don't know the deviation of expert's opinion, so the parameters $(a, b)$ are selected as $(0.3,0.8)$, namely integrate the individual expert opinion under the principal "most". At this point, the initial group decision matrix is obtained.

Group decision making is the process of incorporating the opinion or preference of group expert into a single group opinion or preference under a certain decision criterion [1].This single group opinion should be as far as possible to represent all the experts' preferences, the key is to restrain the influence of expert opinion that has larger deviations. For decision matrix, the difference of expert opinion can be evaluate by deviations between individual expert decision matrix and group decision matrix, the more the deviation degree is, the smaller the weight of the experts is. So the weight of the experts in this paper is the objective weight method. Specific methods are as follows:

Step1. Each row $V_{i}^{k}\left(v_{i 1}^{k}, v_{i 2}^{k}, \cdots, v_{i n}^{k}\right)(i=1,2, \cdots, m)$ of decision matrix represent the opinion of expert $e_{k}$ for the $i$-th program, therefore the difference between each decision matrix can be measured with integrating the deviation by each row firstly, then the differences degree value (namely deviation of decision matrix) between decision matrix can be obtained with integrating deviation of each row in each decision matrix.

If each row of decision matrix is seen as a vector, the deviation between each row of decision matrix can be measured by distance of each vector. In this paper, the distance of vectors is measured with widely used Euclidean distance, which is as follows:

$$
d\left(V_{i}^{c}, V_{i}^{k}\right)=\sqrt{\sum_{j=1}^{n}\left(v_{i j}^{c}-v_{i j}^{k}\right)^{2}}
$$

So the deviation $d\left(V^{c}, V^{k}\right)$ between expert individual decision matrix and group decision matrix based on distance measuring can be defined as follows:

$$
d\left(V^{c}, V^{k}\right)=\frac{\sum_{i=1}^{m} d\left(V_{i}^{c}, V_{i}^{k}\right)}{\sum_{k=1}^{l} \sum_{i=1}^{m} d\left(V_{i}^{c}, V_{i}^{k}\right)}
$$

It is clearly that $d\left(V^{c}, V^{k}\right) \in[0,1]$, the greater the value of $d\left(V^{c}, V^{k}\right)$ is, the greater the deviation between decision matrix is, and the smaller the expert weight is. Expert weight is as follows: 


$$
\beta_{k}=\frac{1-d\left(V^{c}, V^{k}\right)}{\sum_{k=1}^{l}\left[1-d\left(V^{c}, V^{k}\right)\right]} \quad(k=1,2, \cdots, l)
$$

In the type, $\beta_{k} \in[0,1]$.

After the expert individual decision matrix is weighted, the group decision matrix $V^{q}=\left[v_{i j}^{q}\right]_{m \times n}$ can be obtained by using the weighted arithmetic average method, is shown as follows:

$$
v_{i j}^{q}=\sum_{k=1}^{l} v_{i j}^{q} * \beta_{k} \quad(i=1,2, \cdots, m ; j=1,2, \cdots, n)
$$

\section{Numerical Example Analyses}

Assuming that $A=\left\{A_{1}, A_{2}, A_{3}, A_{4}\right\}$ is the program set, $C=\left\{C_{1}, C_{2}, C_{3}, C_{4}\right\}$ is the attribute set, $E=\left\{e_{1}, e_{2}, e_{3}, e_{4}\right\}$ is the expert set, the individual decision matrix from standardized treatment decision matrix given by each expert is as follow:

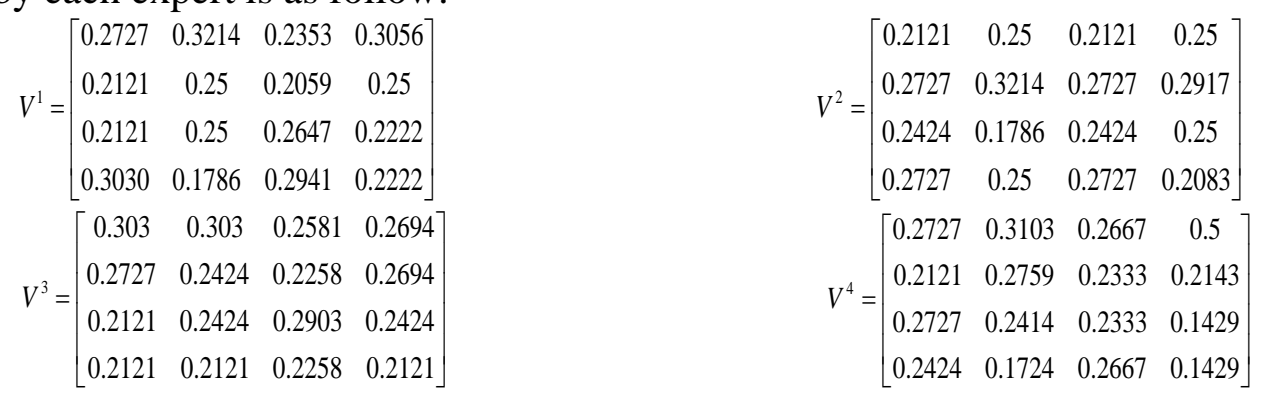

Step1. Deal expert individual decision matrix with OWA operator to obtain the initial group decision matrix $V^{c}$ :

$$
V^{c}=\left[\begin{array}{cccc}
0.2667 & 0.3006 & 0.2421 & 0.2819 \\
0.2363 & 0.2596 & 0.2268 & 0.2650 \\
0.2242 & 0.2355 & 0.2504 & 0.2224 \\
0.2515 & 0.115 & 0.265 & 0.2033
\end{array}\right]
$$

Where, the OWA operator weight vector is $\alpha=(0,0.4,0.5,0.1)^{T}$.

Step2. Analysis the deviation between expert decision matrix and group decision matrix, the distance deviation calculated by Eq.6 is as follow:

$$
\begin{aligned}
& d\left(V^{c}, V^{1}\right)=\frac{0.182}{1.2594}=0.1445 ; d\left(V^{c}, V^{2}\right)=\frac{0.3789}{1.2594}=0.3009 \\
& d\left(V^{c}, V^{3}\right)=\frac{0.2412}{1.2594}=0.1915 ; d\left(V^{c}, V^{4}\right)=\frac{0.4573}{1.2594}=0.3631
\end{aligned}
$$

Step3. Obtain expert weight. Expert weight calculated with Eq.7 is as follow:

$$
\begin{aligned}
& \beta_{1}=\frac{1-0.1445}{3}=0.2852 ; \beta_{2}=\frac{1-0.3009}{3}=0.233 \\
& \beta_{3}=\frac{1-0.1915}{3}=0.2695 ; \beta_{4}=\frac{1-0.3631}{3}=0.2123
\end{aligned}
$$

Step4. Integrate expert individual decision matrix with Eq.8 to obtain group decision matrix, which is shown as follow:

$$
V^{q}=\left[\begin{array}{llll}
0.2667 & 0.2974 & 0.2427 & 0.3242 \\
0.2426 & 0.2701 & 0.2326 & 0.2574 \\
0.2320 & 0.2295 & 0.2597 & 0.2173 \\
0.2586 & 0.2029 & 0.2649 & 0.1994
\end{array}\right]
$$

The group decision matrix constructed is the basis for the follow-up decision-making, the quality of it directly affects the accuracy of result of decision. How to measure the quality of group decision matrix? Currently there is little research on this, and there is no uniformed evaluation criterion.

Looking from the asking of group decision-making result, it is aim to obtain a group opinion that all experts are relatively satisfied, so it can be considered as that if the group matrix obtain by this 
method has a higher comprehensive compliance degree for all of the expert individual decision matrix than the group matrix that obtained by all the others method, group matrix obtained by this method can be considered as a higher quality in some degree. As for how to measure the compliance degree between group decision matrix and expert individual decision matrix, it can be measured by $d\left(V^{q}, V^{k}\right)(k=1,2, \cdots, l), d\left(V^{q}, V^{k}\right)$ calculated with Eq.6, the smaller the $d\left(V^{q}, V^{k}\right)$ value is, the higher the quality of group decision matrix $V^{q}$ is.

Fig. 2 shows the comparison result between method in this article and traditional method, namely geometric mean method, the arithmetic mean method, OWA operator, it is shownThe that method in this article has a higher quality from the degree of compliance between group decision matrix and all the expert decision matrix.

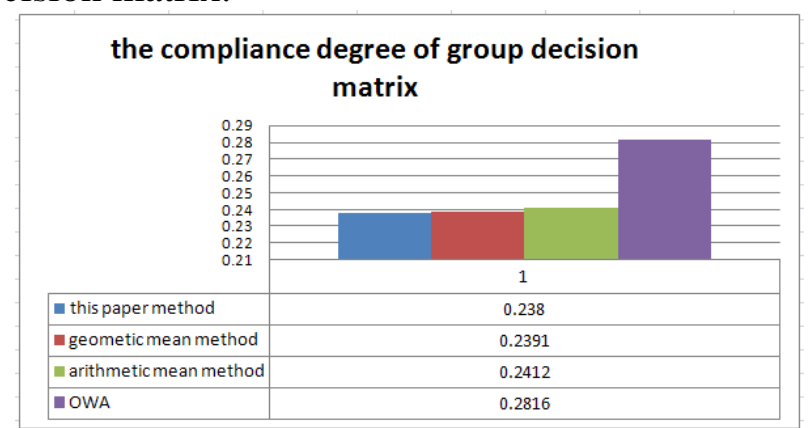

Fig.2. Comparison Result for Group Matrix Constructed by Several Methods

\section{Conclusion}

In this paper, a method for constructing group matrix based on OWA operator has been studied. In the situation of unknown initial expert weight, this method obtains initial group decision matrix by integrating expert individual matrix with OWA operator according to the principle "majority rule", then deviation between group decision matrix and expert individual matrix is obtained with distance measure from initial group decision matrix, therefore to obtain the expert weight, at last the final group decision matrix is obtained by integrating the expert opinion with weighted arithmetic operator.

From the result of numerical example analysis in this article, compared with commonly used information integrating method such as arithmetic mean, geometric mean, OWA operator, method in this article is higher quality in the degree of comprehensive compliance between group decision matrix and expert individual decision matrix.

\section{References}

[1] Zhiping Fan ,Hongyan Li ,Yanping Jiang.Sensitivity Analysis of Group Decision Making Method Based on OWA Operators [J]. Journal of Northeastern University(Natural Science) ,2004 25 (11) 1114-1117.

[2] Krohling R A, Campanharor V C. Fuzzy Topsis for group decision making:A case study for accidents with oil spill in the sea [J] .Expert Systems with Applications,2011 38 (4) 4190-4197.

[3] Wenzhan Dai,Jiuliang Li.Off-target deviation degree method for grey multi-attribute group decision-making [J].Systems Engineering -Theroy \& Practice,2014 34 (3) 787-792.

[4] Youyou Zhang, Jike Lian.A method for determing the weight of expert based on similarity [J].Journal of HENAN Polytechnic University(Natural Science) ,2010 29(2) 283-286.

[5] Junying Wang, Dehua Li. Research on self-adjusting algorithm of group decision-making experts’ weight [J].Application Research of Computer,2011 28 (2) 532-540. 